6. Nseir S, Pronnier P, Soubrier S, Onimus T, Saulnier F, Mathieu D, Durocher A. Fatal streptococcal necrotizing fascitiis as a complication of axillary brachial plexus block. Br J Anaesth 2004; 92 (3): 427.

7. Unglaub F, Guehring T, Fuchs PC, Perez-Bouza A, Groger A. Necrotizing fascitiis following therapeutic injection in a shoulder joint. Orthopade $2005 ; 1: 345$.

8. Saw A, Kwan M K, Sengupta S. Necrotizing fasciitis: a life-threatening complication of acupuncture in a patiente with diabetes mellitus. Singapore Med J 2004; 45 (4): 180-182.

9. Stamenkovic I, Lew PD. Early recognition of potentially fatal necrotizing fasciitis: the use of frozen-section biopsy. N Engl J Med 1984; 310 : 11689-692

10. Dinubile MJ, Lipsky BA. Complicated infections of skin and skin structures: when the infection is more than skin deep. J Antimicrob Chemother 2004; 53: 37-50.

\section{Absceso hepático por Klebsiella oxytoca}

\section{Sr. Director:}

Las bacterias del género Klebsiella son bacilos gram-negativos que se caracterizan por poseer una prominente cápsula de polisacáridos. En la actualidad se considera que existen tres especies de estos microorganismos: Klebsiella pneumoniae, K. oxytoca y $K$. variicola (1).K. pneumoniae es el principal agente patógeno del grupo, mientras que las otras especies producen infecciones con mucha menor frecuencia. Describimos a continuación un caso de absceso hepático producido por $K$. oxytoca.

Paciente de 73 años con antecedentes de diabetes mellitus tipo 2, hipertensión arterial, claudicación intermitente de los miembros inferiores, colecistectomía, y esfinterotomía por colangitis secundaria a litiasis biliar. Ingresa por fiebre y escalofríos que no ceden tras tres días de tratamiento con eritromicina. A la exploración presenta buen estado general, temperatura axilar $39^{\circ} \mathrm{C}$, auscultación cardiaca y pulmonar normal, abdomen blando y depresible con signos de Murphy y de Blumberg negativos, pulsos pedios disminuidos y ausencia de focalidad neurológica.

Los análisis muestran leucocitos $7.250 / \mathrm{mm}^{3}$ con $78 \%$ de segmentados, hemoglobina $12,6 \mathrm{~g} / \mathrm{dl}$, plaquetas $162.000 / \mathrm{mm}^{3}$, glucosa $256 \mathrm{mg} / \mathrm{dl}$, urea $43 \mathrm{mg} / \mathrm{dl}$, creatinina $1,44 \mathrm{mg} / \mathrm{dl}$, bilirrubina 1,16 mg/dl, proteína C reactiva $102 \mathrm{mg} / \mathrm{dl}$, GOT 56 UI/l, GPT 63 UI/l, y amilasa y coagulación normales. La serología es normal. $\mathrm{El}$ análisis de orina es normal, y el urinocultivo es negativo. Los hemocultivos son positivos para Klebsiella oxytoca. Las radiografías de tórax y de abdomen y el electrocardiograma son normales. La ecografía abdominal muestra aerobilia con una vía biliar no dilatada. El TAC abdominal pone de manifiesto un absceso en el segmento antero-superior del lóbulo hepático derecho, de contorno irregular y de unos $4 \mathrm{~cm}$ de diámetro máximo. Durante los primeros días de su estancia presenta varios picos febriles con escalofríos; coincidiendo con uno de ellos sufre un episodio de pérdida de conciencia y convulsiones tónico-clónicas generalizadas, del que se recupera espontáneamente; el electroencefalograma es normal. Una vez establecido el diagnóstico de absceso hepático se inicia tratamiento con ciprofloxacino y metronidazol. Al recibirse el resultado de los hemocultivos se continúa el tratamiento con ciprofloxacino, que se mantiene 8 semanas; el TAC de control realizado dos meses después muestra la resolución del absceso. Tres años más tarde el paciente sigue asintomático.

El absceso piogénico hepático es un tipo de infección poco frecuente. Se trata de un proceso grave, que hasta hace pocos años presentaba una mortalidad superior al $50 \%$. No obstante, en la actualidad, su pronóstico ha mejorado substancialmente gracias al desarrollo de los procedimientos diagnósticos de imagen y a la mayor disponibilidad de tratamientos eficaces, como el drenaje percutáneo (2).

Esta enfermedad puede estar producida por numerosos gérmenes, pero tradicionalmente se ha considerado a Escherichia coli como el principal agente responsable. Los abscesos hepáticos por Klebsiella spp. se describieron inicialmente en los países orientales (3). Pero en los últimos años, se han comunicado casos de dicha infección en varios países occidentales, entre ellos en España (4), y además, según algunos estudios $(2,5)$, su incidencia está aumentando progresivamente.

La inmensa mayoría de abscesos hepáticos por Klebsiella spp. están producidos por $K$. pneumoniae $(2,5)$. En una revisión de la literatura de los últimos 25 años por medio de la base de datos Medline (perfil de búsqueda: (liver OR hepatic) AND abscess AND oxytoca), sólo hemos encontrado descrito otro caso de dicha infección producido por $K$. oxytoca, similar al nuestro (6). En ambos casos el absceso hepático ocurre en pacientes con diabetes, se presenta con un proceso febril de causa no evidente, únicamente se logra establecer el diagnóstico al realizar estudios de imagen, y se obtiene una buena respuesta con tratamiento antibiótico y drenaje del absceso.

\section{B. Roca, D. Ferrer, A. P. Pérez}

Unidad de Enfermedades Infecciosas. Hospital General. Castellón

1. Martínez J, Martínez L, Rosenblueth M, Silva J, Martínez-Romero E. How are gene sequence analyses modifying bacterial taxonomy? The case of Klebsiella. Int Microbiol 2004; 7: 261-268.

2. Rahimian J, Wilson T, Oram V, Holzman RS. Pyogenic liver abscess: recent trends in etiology and mortality. Clin Infect Dis 2004; 39: 1654 1659.

3. Yang CC, Yen CH, Ho MW, Wang JH. Comparison of pyogenic liver abscess caused by non-Klebsiella pneumoniae and Klebsiella pneumoniae. J Microbiol Immunol Infect 2004; 37: 176-184.

4. Tordera P, Blanes M, Cercós A, Salavert M, Velasco J, López J. Abscesos hepáticos recidivantes por Klebsiella pneumoniae. Rev Clin Esp 2003; 203: 310-312.

5. Lederman ER, Crum NF. Pyogenic liver abscess with a focus on Klebsiella pneumoniae as a primary pathogen: an emerging disease with unique clinical characteristics. Am J Gastroenterol 2005; 100: 322-331.

6. Saad F, Ach K, Dallel Youssef N, Maarouf A, Chaieb Chadli M, Chaieb L. Abces hepatique chez le diabetique. 2 observations. Presse Med 2004; 33: $98-100$.

\section{¿Cómo se presenta la espondilitis infecciosa?}

\section{Sr. Director:}

Hemos leído con interés el artículo publicado en su revista por Gómez Rodríguez y cols. (1) en el que realizan un análisis retrospectivo de las espondilitis infecciosas diagnosticadas en su área durante 20 años. Nos parece de interés comentar en este apartado, y a propósito de este artículo, un trabajo en el mismo sentido que nosotros hemos realizado recientemente en nuestro hospital, con la intención de poder aportar alguna información más sobre este tema ya que, al no ser una patología muy frecuente, el número de pacientes incluidos en las series suele ser muy pequeño.

En nuestro estudio revisamos las historias clínicas que incluyeron este diagnóstico en los últimos 5 años en nuestro hospital. 
Encontramos 27 casos, de los cuales el 63\% eran varones, con una edad media de 58,6 $\pm 19,4$ años. En un 70,4\% de los casos se aisló el germen responsable siendo la causa tuberculosa la más frecuente $(42 \%)$ seguida del Staphylococcus spp con un $37 \%$ de los pacientes. Entre los factores de riesgo estudiados el que más veces se veía relacionado era la presencia de una infección concomitante (Tabla I). Todos los pacientes presentaban dolor mecánico que se irradiaba a miembros inferiores en la mitad de ellos. Además se constató fiebre en el $56 \%$, síntomas neurológicos en el $42,3 \%$, rigidez en el $15,4 \%$ y síndrome constitucional en sólo el $11,5 \%$. Se tardó una media de 57,7 $( \pm 53,7)$ días en llegar al diagnóstico y la estancia media en el hospital fue de 38,2 ( $\pm 23,7)$ días. Con respecto a los parámetros analíticos, existía leucocitosis en el 53,8\% de los casos y una velocidad de sedimentación elevada (VSG) en el 95,7\%. Por regiones, la localización lumbar fue la más frecuente $(55,6 \%)$. En el 79,3\% de los pacientes con radiografía, ésta era patológica. En 25 de los 27 casos se llegó al diagnóstico mediante resonancia magnética nuclear (RM) $(96,2 \%)$. Todos los pacientes recibieron tratamiento antibiótico precisando cirugía el 33,3\%. Concluyendo: la espondilitis infecciosa es una patología poco frecuente por lo que, a la dificultad de su diagnóstico, se une la falta de sospecha por parte del facultativo. Ello explicaría el retraso de su diagnóstico y la alta estancia hospitalaria. En nuestra serie, y a pesar de ser más actual en el tiempo, aparece la etiología tuberculosa como la primera causa por delante de Staphylococcus. Nosotros no analizamos la evolución de las distintas etiologías en el tiempo como han hecho

\section{TABLA I}

\begin{tabular}{lc}
\multicolumn{2}{c}{ FACTORES DE RIESGO RECOGIDOS PARA ESPONDILITIS } \\
INFECCIOSA
\end{tabular}

Gómez Rodríguez y cols. (1), por lo que no podemos saber si en los años precedentes los casos tuberculosos fueron todavía más numerosos que actualmente; únicamente podemos afirmar que en nuestra área que es una zona urbana, la tuberculosis sigue siendo la causa más frecuente de espondilodiscitis a pesar de las mejoras en el diagnóstico y tratamiento de esta patología y que se apuntan como responsables en el artículo referido. La presencia de espondilodiscitis parece estar relacionada con una cirugía sobre la columna o una infección en otra localización previas; también hemos recogido otros factores predisponentes como la diabetes y las neoplasias pero que no han sido los que más se han relacionado con el desarrollo de la infección. Todos los pacientes debutan con dolor siendo más inconstantes otros síntomas como la fiebre o el síndrome constitucional. La VSG es el marcador analítico más importante para el diagnóstico y seguimiento, siendo la leucocitosis un parámetro más inconstante. En general los facultativos preferimos la RM para su detección siendo la localización lumbar la más frecuente.

El diagnóstico de espondilitis infecciosa será siempre algo inusual para el médico pero no por ello de menor importancia. Cuando no existen predisponentes claros o una clínica orientativa es un diagnóstico difícil que muchas veces se retrasa conllevando un alto coste sanitario y sobre todo personal. Su conocimiento entonces debe pasar a dejar de ser una mera anécdota para el internista. Por último, cabe reseñar que todas nuestras conclusiones se extraen de la descripción de los casos que hemos encontrado en nuestro centro, pero aún así creemos que se necesitan nuevos estudios en los que se puedan incluir mayor número de pacientes.

\section{E. Álvarez-Rodríguez, R. Torres-Gárate, A. Gutiérrez Larráinzar, M. Serrano Cazorla, L. Mao, M. A. Lozano Ton- kín}

Servicio de Medicina Interna I. Hospital Clínico San Carlos. Madrid

1. Gómez Rodríguez N, Penelas-Cortés Bellas Y, Ibáñez Ruán J, González Pérez M, Sánchez Lorenzo ML. Espondilodiscitis infecciosas en un área sanitaria gallega, 1983-2003. An Med Interna (Madrid) 2004; 21: 53339. 